\title{
Challenges and opportunities of noninvasive cardiac imaging in obesity
}

\author{
Alberto Cuocolo, $\mathrm{MD}^{\mathrm{a}}$ \\ ${ }^{a}$ Department of Advanced Biomedical Sciences, University of Naples Federico II, Naples, Italy
}

Received Sep 9, 2016; accepted Sep 9, 2016

doi: 10.1007/s12350-016-0676-z

Numerous evidences support obesity as both an independent risk factor and a risk marker for the development of asymptomatic and symptomatic coronary artery disease (CAD), heart failure, and atrial fibrillation. ${ }^{1}$ Diagnosis and risk stratification of cardiovascular impairment in obese population are important to identify those subjects who might benefit from more straight therapeutic strategies. Yet, noninvasive cardiac imaging techniques have restraints in obese subjects and the diagnostic accuracy is often limited. When physical exercise is used as stressor, the poor exercise tolerance leads to underestimation of inducible ischemia. The poor acoustic window for ultrasound in patients with obesity affects the performance of echocardiography in depiction of global and regional motion, thickness, and function of left ventricle. The excess soft tissue attenuation of radioactivity frequently produces artifactual myocardial perfusion defects and poor signal-to-noise ratios for single-photon emission computed tomography (SPECT) imaging. ${ }^{2}$ The value of attenuation correction is easy to accept, and it is generally recognized that it is important to discern if a tracer deficit is due to diminished myocardial perfusion or if it is an attenuation or motion artifact. Another problem is to establish whether the defect is really reversible or represents a result of scatter and interference from extracardiac tracer activity. It seems conceivable that attenuation correction should at least improve the problems related with an attenuation artifact. However, the question of whether attenuation correction is needed is still unsolved and it has been debated for a long time. ${ }^{3}$ Gantry bore and table weight limits also affect the ability to accommodate high grade obese on conventional SPECT or positron emission tomography (PET). Hence, it is critical to identify

\footnotetext{
Reprint requests: Alberto Cuocolo, MD, Department of Advanced Biomedical Sciences, University of Naples Federico II, Via Pansini 5, 80131 Naples, Italy; cuocolo@unina.it

J Nucl Cardiol 2016;23:1233-4.

$1071-3581 / \$ 34.00$

Copyright (C) 2016 American Society of Nuclear Cardiology.
}

noninvasive imaging modalities with preserved diagnostic and prognostic values irrespective of inpatients' body mass index (BMI).

New pharmacological stressors and most recent hardware and software implementation of available noninvasive imaging techniques paved the way to the opportunity of studying obese subjects. Pharmacological stress imaging might be appropriate for subjects with inability to exercise. Regadenoson is a selective adenosine $\mathrm{A}_{2 \mathrm{~A}}$ receptor agonist with many of the characteristics of an ideal stress perfusion agent, being a potent and a selective coronary vasodilator with a rapid onset of action, a short duration of action, and being administered as a fixed-dose bolus (not weight-based). It can be safely administered in all patient categories with a great efficacy regardless of BMI. This drug is better tolerated than adenosine, overcoming limitation related to weight-adjusted dosing suggesting a potential benefit in using this stressor instead of conventional pharmacological stressors. ${ }^{4}$ PET/CT imaging allows accurate attenuation correction, reducing false positive results in obese subjects. An increase in body weight is also independently associated with abnormal coronary circulatory function that progresses from an impairment in endothelium-related coronary vasomotion in overweight individuals to an impairment of the total vasodilator capacity in obese individuals. ${ }^{5}$ Further, gastric bypassrelated reduction of BMI in morbidly obese individuals beneficially affects coronary circulatory dysfunction. ${ }^{6}$ The use of myocardial perfusion PET may increase with the introduction of new ${ }^{18} \mathrm{~F}$-fluorinated perfusion compounds that are currently under evaluation. Recent preliminary results of Phase 3 multicenter trial demonstrates superiority of flurpiridaz over SPECT for the assessment of CAD in a large population of obese subjects, accompanied by significantly lower radiation exposure. ${ }^{7}$ The assessment of myocardial perfusion and coronary blood flow with new stressors and tracers may lead to a more effective personalized, image-guided therapy approach that may offer potential to further 
improve clinical outcome also in obese patients. ${ }^{8}$ Ultrafast high-efficiency SPECT cameras with cadmium-zinc-telluride (CZT) semiconductor detectors have been recently introduced. These systems improve the overall sensitivity of SPECT method showing highlevel performance for detection of CAD also in patients with excess adiposity ${ }^{9}$ and allow easily positioning, with a weight limit of $246 \mathrm{~kg}$ (vs $180 \mathrm{~kg}$ for conventional SPECT systems). A ten-second pre-scan acquisition permits identification of heart location, even in obese subjects in whom the heart is positioned far from the surface of the chest wall. Feasibility of coronary flow reserve estimation by CZT SPECT cameras may open up to the opportunity to investigate coronary reserve even in obese population that is not eligible to absolute quantification of myocardial blood flow using PET/CT ${ }^{10}$ allowing more large-scale investigations. Obesity has also a significant impact on I-123 metaiodobenzylguanidine indexes of cardiac sympathetic innervation in patients with heart failure, suggesting a potential role of excess adiposity in worsening sympathetic innervation. ${ }^{11}$ Thus, not only the detection of CAD but also the evaluation of early adrenergic dysfunction might improve clinical assessment of subjects with excess adiposity. Future studies to answer the question of which imaging technique may offer the optimal approach in obese patients are urgently needed.

\section{Disclosure}

The author has indicated that he has no financial conflict of interest.

\section{References}

1. Mandviwala T, Khalid U, Deswal A. Obesity and cardiovascular disease: A risk factor or a risk marker? Curr Atheroscler Rep 2016;18:21.
2. Lim SP, Arasaratnam P, Chow BJ, Beanlands RS, Hessian RC. Obesity and the challenges of noninvasive imaging for the detection of coronary artery disease. Can J Cardiol 2015;31:223-6.

3. Cuocolo A. Attenuation correction for myocardial perfusion SPECT imaging: Still a controversial issue. Eur J Nucl Med Mol Imaging 2011;38:1887-9.

4. Cerqueira MD, Nguyen $P$, Staehr P, Underwood SR, Iskandrian AE, ADVANCE-MPI Trial Investigators. Effects of age, gender, obesity, and diabetes on the efficacy and safety of the selective A2A agonist regadenoson versus adenosine in myocardial perfusion imaging integrated ADVANCE-MPI trial results. JACC Cardiovasc Imaging 2008;1:307-16.

5. Schindler TH, Cardenas J, Prior JO, Facta AD, Kreissl MC, Zhang $\mathrm{XL}$, et al. Relationship between increasing body weight, insulin resistance, inflammation, adipocytokine leptin, and coronary circulatory function. J Am Coll Cardiol 2006;47:1188-95.

6. Quercioli A, Montecucco F, Pataky Z, Thomas A, Ambrosio G, Staub C, et al. Improvement in coronary circulatory function in morbidly obese individuals after gastric bypass-induced weight loss: Relation to alterations in endocannabinoids and adipocytokines. Eur Heart J 2013;34:2063-73.

7. Bateman TM, Maddahi J, Udelson J, Beanlands R, Knuuti J, Heller G, et al. Improved assessment of CAD in obese subjects with flurpiridaz F18 PET myocardial perfusion imaging: A subset analysis of the flurpiridaz F18 301 phase 3 study. J Am Coll Cardiol 2016;67:1578.

8. Schindler TH. Positron-emitting myocardial blood flow tracers and clinical potential. Prog Cardiovasc Dis 2015;57:588-606.

9. Nakazato R, Tamarappoo BK, Kang X, Wolak A, Kite F, Hayes $\mathrm{SW}$, et al. Quantitative upright-supine high-speed SPECT myocardial perfusion imaging for detection of coronary artery disease: Correlation with invasive coronary angiography. J Nucl Med 2010;51:1724-31.

10. Petretta M, Storto G, Pellegrino T, Bonaduce D, Cuocolo A. Quantitative assessment of myocardial blood flow with SPECT. Prog Cardiovasc Dis 2015;57:607-14.

11. Pellegrino T, Piscopo V, Boemio A, Russo B, De Matteis G, Pellegrino S, et al. Impact of obesity and acquisition protocol on (123)I-metaiodobenzylguanidine indexes of cardiac sympathetic innervation. Quant Imaging Med Surg 2015;5:822-8. 